\title{
Controlling for loT Projects Systems based on Smart Phone using Firebase Notification Technique
}

\author{
Abbas Mohammed Younus Yousif \\ Iraq-Najaf
}

\begin{abstract}
Internet of Things (IoT) projects have become very popular in the fields of information technology and spread widely among technicians and engineers. These projects play an important role in our daily life, especially after the emergence of new technologies that have made these projects more flexible and close to the needs of the human community and also when cloud computing started to provide services to these projects. In this paper I shed light on one of those techniques provided by the multinational technology company (google), which is a (firebase) technology that provides many services for projects whether small project or large, including database service, license service and notification service etc., In this paper I will provide a detailed explanation of notification service over IoT. This work will implement in two parts: Hardware which consist of Arduino, a ESP8266 Wi-Fi controller, and a keypad that use to access to the system when typing the secret code, and software include android application that contact with firebase. When a new user turn on the device, it will send notify to the smart phone using firebase notification to alert someone tried to access to the system. The owner will resend notify to device to give permission and store it in firebase database or prevent the user which the keypad will be disable.
\end{abstract}

\section{Keywords}

Arduino, ESP8266, Firebase, Android Studio.

\section{INTRODUCTION}

The Internet of Things (IoT) is the over networking of the devices, vehicles (also referred to as "connected devices" and "smart devices"), buildings, everything. with electronics, software, sensors, and network connectivity which enable these items to contact among them and exchange data[1]. The IoT provide sense to objects or controlled remotely across existing network infrastructure, but building an IoT application needed the selection and integration of multiple components: sensors, communications issues, and networks as a start. And turning the data that you collect into something useful will typically involve cloud computing, analytics, integration with core systems, and process changes [2]. The integration of other technologies with IoT project makes the project more develop and expanded in the services provided, and what has been done in this paper shows the importance of using cloud technology like firebase to provide system protection which used such as notify transition medium between arduino device and smart phone.

\section{HARDWARE PART}

This part contain microcontroller (in this project used Arduino) and some peripheral components like 4x4 keypad use to type user name and password to input to the system, and ESP8266 WiFi Module that can give any microcontroller access to WiFi network and green LED that light when system open and red LED light when system is close, also other components require to connect all of among them. In addition there is mobile phone with android OS to receive alert from arduino. (Figure.1) display whole connecting project:

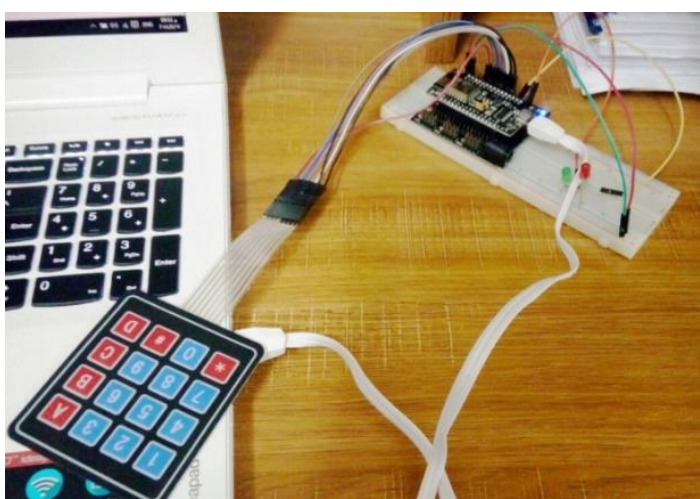

Fig.1: The project device

I will take a brief explanation of each important components in this part:

\subsection{Arduino UNO}

The Arduino microcontroller is an easy to use yet powerful single board computer that has gained considerable traction in the hobby and professional market. The Arduino is opensource [3]. There are various types of Arduino, the type that used in this project is Arduino Uno which has 14 digital input/output pins (of which 6 can be used as PWM outputs), 6 analog inputs, a $16 \mathrm{MHz}$ quartz crystal, a USB connection, a power jack, an ICSP header and a reset button.( See Figure.2).

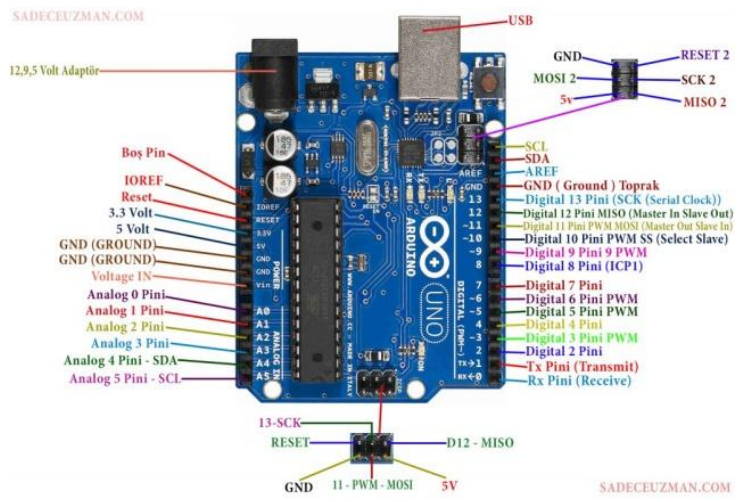

Fig. 2: Arduino Uno board.

It contains everything needed to support the microcontroller; simply connect it to a computer with a USB cable or power it with a AC-to-DC adapter or battery to get started. Also can tinker with UNO without warring too much about doing something wrong, worst case scenario which can replace the chip for a few dollars and start over again.

\subsection{ESP8266 Chip}

The ESP8266 is WiFi Module with full TCP/IP protocol stack that can give any microcontroller access to the WiFi network. ESP8266 is a complete and self-contained Wi-Fi network solution that can carry software applications, or through another application processor uninstall all Wi-Fi networking capabilities. ESP8266 when the device is mounted and as the 
only application of the application processor, the flash memory can be started directly from an external Move. Builtin cache memory will help improve system performance and reduce memory requirements [4]. Another case is when wireless Internet access assume the task of Wi-Fi adapter, In addition it can be added to any microcontroller-based design.( See Figure.3).
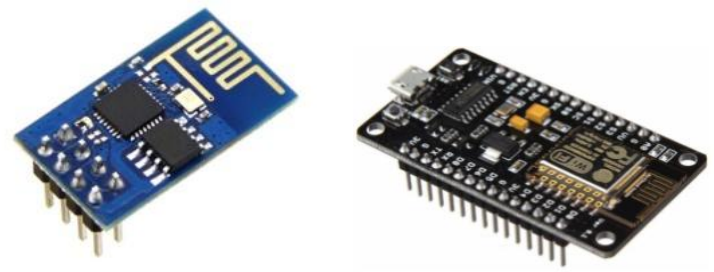

Fig. 3: ESP8266 Chips

\subsection{Keypad}

The Keypad library allows your Arduino to read a matrix type keypad. They come in $3 \times 4,4 \times 4$ and various other configurations with words(See Figure.4), letters and numbers written on the keys. This library is capable of supporting all of those.

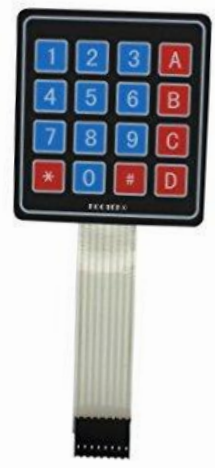

Fig. 4: 4x4 Keypad.

Connecting of main components of the device is implemented in table. 1 which describe connect of each pin in component and Arduino:

Table.1. Detail of components connecting.

\begin{tabular}{|c|c|c|}
\hline Pins & Components & Details \\
\hline 0 & Esp8266 & $\mathbf{R x}$ \\
\hline 1 & Esp8266 & $\mathbf{T x}$ \\
\hline 2 & \multirow{8}{*}{ Keypad } & row_1 \\
\hline 3 & & row_2 \\
\hline 4 & & row_3 \\
\hline 5 & & row_4 \\
\hline 6 & & colomn_1 \\
\hline 7 & & colomn_2 \\
\hline 8 & & colomn_3 \\
\hline 9 & & colomn_4 \\
\hline 10 & LED & red \\
\hline 11 & LED & green \\
\hline
\end{tabular}

\section{SOFTWARE PART}

It's important part which is the soul of the hardware components and controls the work for each one and data streaming among them, this part consist of three major software: Arduino IDE, Firebase console and Android Studio.

\subsection{Arduino IDE}

The Arduino Integrated Development Environment is special software running on our computer that we can write sketches for the Arduino board. (See Figure.5). And then we will upload the sketch to the board, the code that we have written is compiled into low level language that is understood by the microcontroller [5]. This last step is quite important, because it's where Arduino makes our life simple by hiding away as much as possible of the complexities of programming microcontrollers.

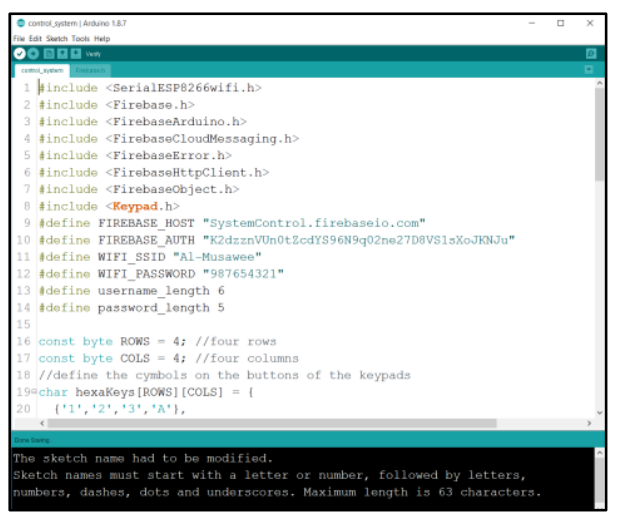

Fig. 5: The Arduino IDE's.

Here should be add firebase and esp8266 wifi library to enable device to connect to internet and firebase.

\subsection{Firebase Console}

Firebase provide power to application backend, including data storage, user authentication, static hosting, notification and more (See Figure.6).

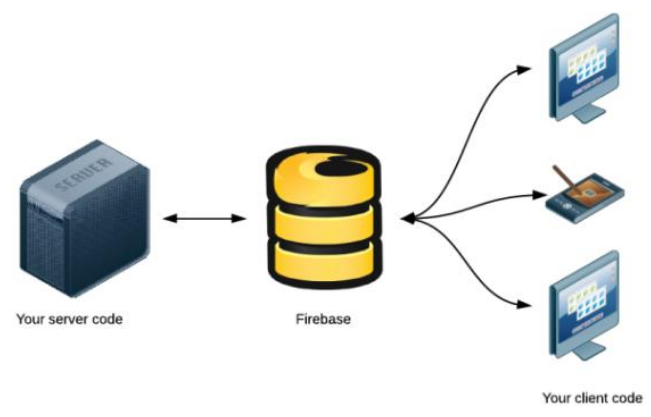

Fig. 6: server and client connection via firebase.

Focus on creating extraordinary user experiences, It's provide tools to develop high-quality web and smart phone applications. Firebase used to send notify to the mobile device application after receive it from Arduino in addition it will store user name and password.

\subsubsection{Firebase Notification Sending}

This process done by using Firebase Cloud Messaging (FCM), which is a cross platform messaging that allow us reliably send messages to the applications.

By using FCM, we can send notification to client application that new email or other data is available to synchronize. For 
use cases such as instant messaging, a message can transfer a payload of up to $4 \mathrm{~KB}$ to a client application [6].

\subsection{Android Studio}

The project that introduce in this paper use android operating system application to receive alert from firebase (Although it's possible to use cross platform for all OS's), so it's needed an IDE to create application. The best environment use for this purpose is android studio in general because it's supported by google company and contains large of libraries and tools that help the programmer in code and design easily, also I can add firebase api to the project easily. (See Figure.7)

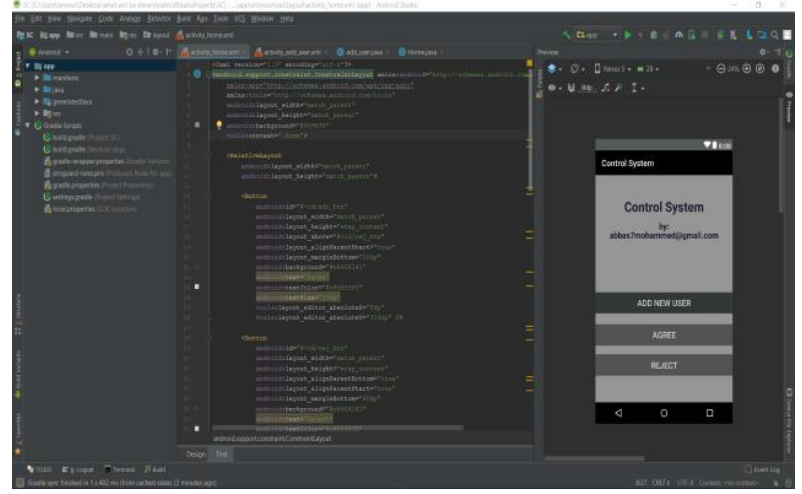

Fig. 7: Android studio platform.

Android Studio is designed specifically for Android development. It is provided on Windows, Mac OS X and Linux, and replaced Eclipse Android Development Tools (ADT) as Google's primary IDE for native Android application development [7]

\section{PROPOSED WORK}

Proposed system diagram implemented in (Figure.8) which describe work of the system and how the data translating form user to Arduino chip and how its exchange between owner smart phone and cloud server.

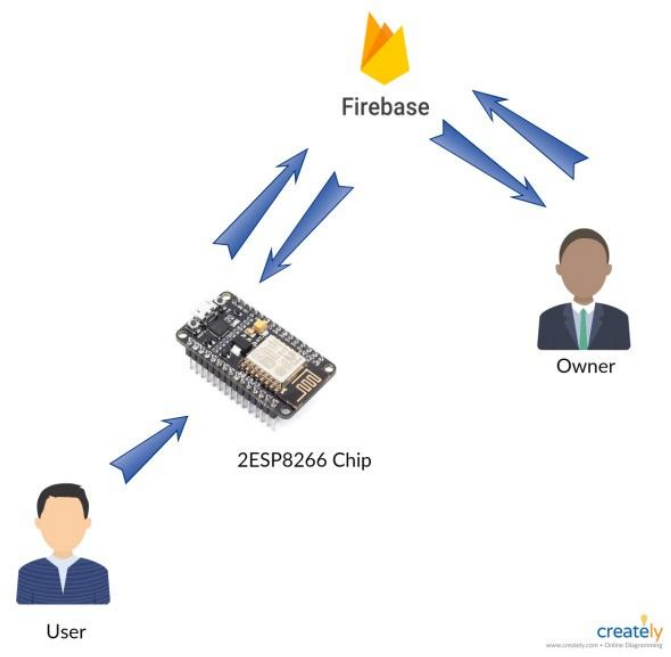

Fig. 8: Proposed System Implementation.

The following flowchart that shown in (Figure.9) shows the structure of system work, which start by inserting user name and password from user to device (Arduino) and it will be check if this user exist in firebase or not.

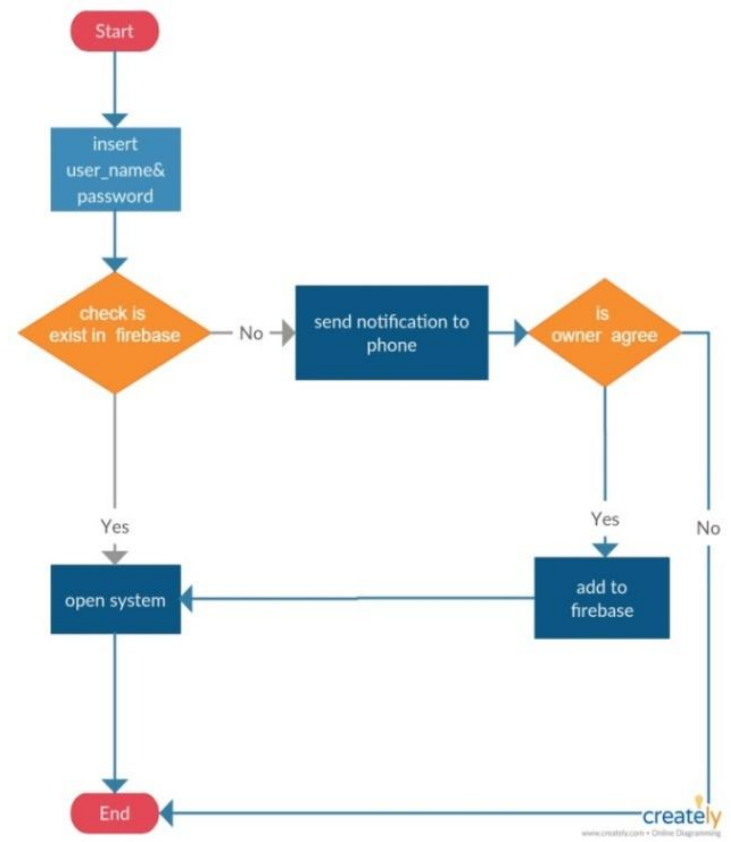

Fig. 9: Project Flowchart.

So if its exist then the system will be open (green LED will light) otherwise it will send notification to phone application to tell owner that a new user try to reach to the system. (See Figure.10)

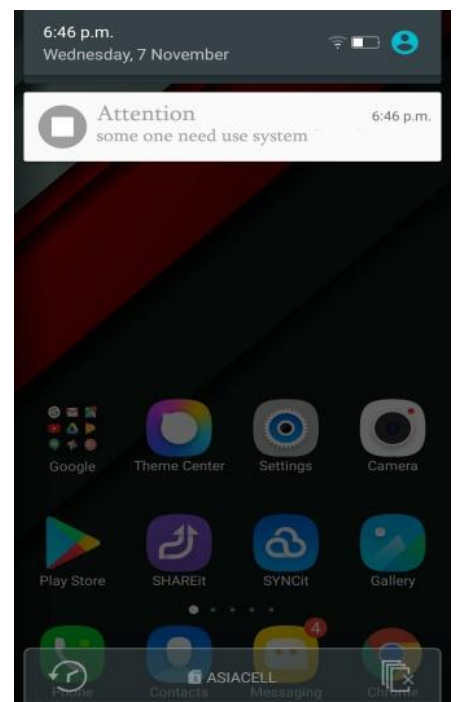

Fig. 10: Receiving Notification from Arduino.

Therefor if owner give accept (click AGREE), this user add to firebase database and the value of status field in firebase will be "1" where lead system to opening, and if he not accept (click REJECT) it will be close (red LED will light)(See Figure.11). 


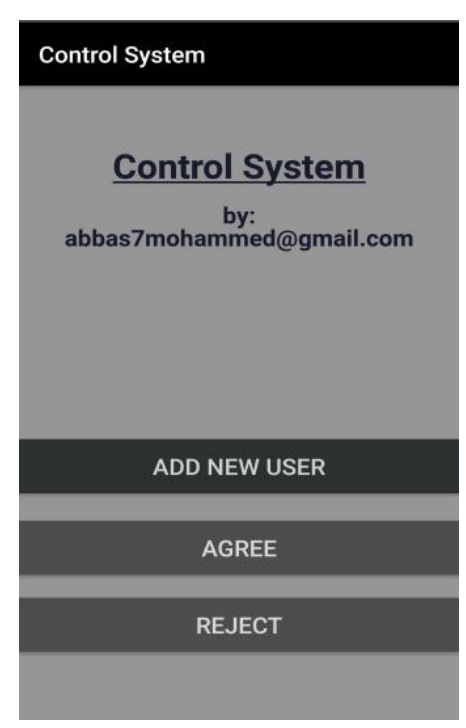

Fig. 11: Application User Interface.

This operation will be done by firebase cloud messaging. In (Figure.12) shown capture of firebase database, as its seem there is one user stored and its password.

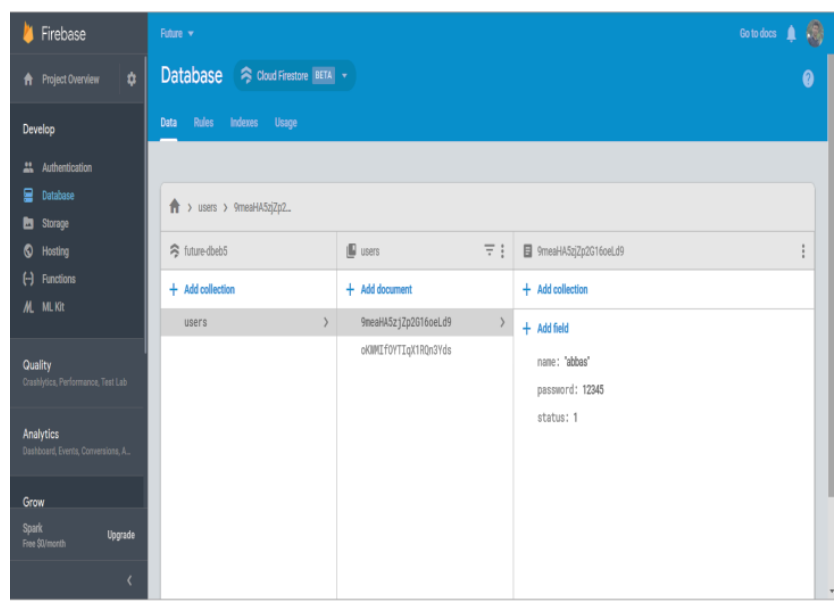

Fig. 12: Firebase Database.

In addition the phone application allow to add more users to system by clicking to Add button which open new window and it ask to input user name and password the by click add it will storage in firebase and the value of status field will be "1" automatically (See Figure.13).

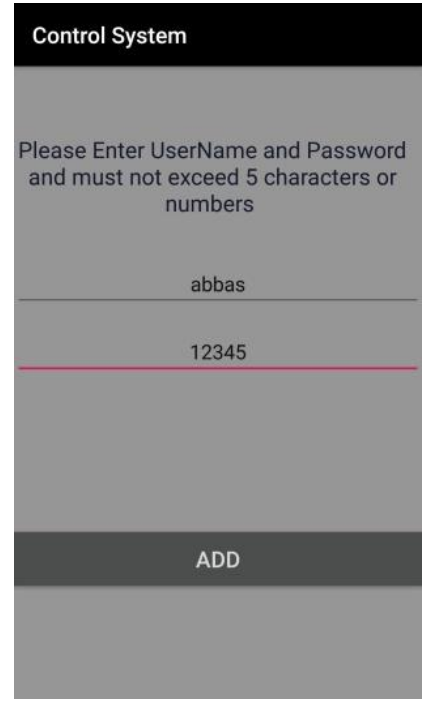

Fig. 13: New user adding layout.

\section{CONCLUSION}

It is very important to insert cloud computing into the projects. This technology provides a wide range of services that facilitate the work of projects especially the remote control of the project, where the person can access on his project from anywhere in the world and control it easily.

In this paper I tried to present a small model of those projects and how to control it by using firebase technique which provide database service and cloud messaging. The person can control to his system device remotely by send agree or reject to the device across firebase.

\section{REFERENCES}

[1] Brown, Eric (13 September 2016). "Who Needs the Internet of Things?". Linux.com.

[2] Mark Bartolomeo (23 October 2016) "Internet of Things: Science Fiction or Business Fact?" Harvard Business Review.

[3] W. Durfee, (oct-2011),"Arduino Microcontroller Guide", University of Minnesota.

[4] Max Filippov, (Mar 6, 2015), "ESP8266 Serial Esp-01 WIFI Wireless".

[5] Massimo Banzi, (2011), "Getting Started with Arduino",U.S.A

[6] https://firebase.google.com/docs/cloud-messaging/

[7] Singh, S. Sharma and S. Singh "Android Application Development using Android Studio and PHP Framework", International Journal of Computer Applications, U.S.A. 